\title{
Synthesis of Equilibrium and Non-Equilibrium Thermodynamics
}

\author{
V.A. Etkin \\ Togliatti State University, Togliatti, Russian Federation. \\ E-mail: etkin.v@mail.ru
}

\begin{abstract}
The possibility of synthesis of equilibrium and non-equilibrium thermodynamics (thermostatics and thermokinetics) on classical principles as a deductive and phenomenological theory has been substantiated. This is achieved in the article on the basis of the law of conservation of energy due to its expression through the flows of the energy carrier and the introduction of the missing parameters of the nonequilibrium state of the system. The proposed way of constructing nonequilibrium thermodynamics as a consequence of energodynamics makes it possible to substantiate all the provisions of the theory of irreversible processes (TIP) and classical thermodynamics without involving hypotheses, postulates and considerations of a molecular-kinetic and statistical-mechanical nature. This approach prevents the occurrence of thermodynamic inequalities and opens up the possibility of generalizing TIP to the processes of useful energy conversion, primarily of interest to power engineers, technologists, biophysicists and astrophysicists.
\end{abstract}

Received 9 April 2021, Revised 24 May 2021, Accepted 27 July 2021

Keywords: Thermodynamics; kinetics; irreversibility; conservation laws; energy transfer and conversion processes; reciprocity relations; superposition effects.

\section{Introduction.}

When they talk about a revolution in physics of the twentieth century, they usually mean quantum mechanics (QM) and the theory of relativity (SRT and GRT). Meanwhile, along with them in the first third of the same century, another no less revolutionary theory arose - the thermodynamics of irreversible processes (TIP). It arose on the basis of the pioneering works of the future Nobel Prize winner Dane Lars Onsager as a theory of the rate of relaxation processes, which he called "quasithermodynamics" [1]. Although the subject of her research - relaxation processes - fell within the competence of classical thermodynamics [2], this theory proposed a method for describing the kinetics of such processes. She returned to thermodynamics the concepts of the driving force and the rate of a process, which had lost their meaning for quasi-static processes, and thereby gave thermodynamics the ability to determine the causes of occurrence, the direction and speed of their course. This theory did not exclude the possibility of a number of processes that contradict classical thermodynamics, such as "active transport" and "upward diffusion" (transfer of matter in the direction of increasing its concentration, "coupled" cyclic Belousov-Zhabotinsky reactions, and "self-organization" of systems far from equilibrium [3] This contradicted the beliefs of A. Einstein, who considered classical thermodynamics "the only theory of general content, the consequences of which will never be refuted by anyone." [4] Therefore, TIP can be considered a theory no less revolutionary than QM and GTR.

Thus, in the first third of the twentieth century, three new theories came to the fore. Each of these theories had its own conceptual system, its own mathematical apparatus and its own model ideas about the structure of matter. Each was based on a number of additional hypotheses and postulates, some of which, in principle, could not be confirmed by experience with the existing level of experimental and observational tools. Therefore, they developed independently, having practically no points of contact. The disagreements between them were so great that the synthesis of at least two of the first of them - QM and GTR - was assessed by A. Einstein as "Great Unification". There has come a stagnation, which most scientists call the crisis of theoretical physics as a whole, and at best, the "crisis of its misunderstanding."

It is of interest to find out what role thermodynamics played in this respect and what caused such a sudden rejection of classical concepts by physicists? After all, it was precisely the theory of thermal radiation that was one of the main reasons for the crisis that led to the replacement of classical mechanics with quantum mechanics. And it was in it that a "glaring contradiction" arose with the nature of the evolution of biological systems [2], as well as the theory of "thermal death" of the Universe, which has not yet received confirmation [3]. On the other hand, it was the classical thermodynamics, which operated with the concepts of absolute temperature, pressure and entropy and did not need inertial reference frames (IRF), that should have prevented the emergence of the theory of relativity.

The author of this article has devoted at least 50 years of his creative life to clarifying this issue, a doctoral dissertation [5], a number of monographs [6-9] and many articles, some 
of which are collected in the book [10]. Its goal was to identify and, if possible, eliminate those obstacles that seemed insurmountable at the beginning of the twentieth century. This article will summarize these studies, which led to the development of energodynamics [8] as a unified theory of the processes of transfer and conversion of thermal energy, including, as special cases, the classical thermodynamics of R. Clausius and "quasithermodynamics" of L. Onsager.

\section{Methodological features of energodynamics}

Like classical thermodynamics, energodynamics is built as a consistently deductive and phenomenological discipline, which is alien to the use of model concepts of the microscopic "mechanism" of processes, as well as hypotheses and postulates in the foundations of the theory ${ }^{1}$. In it, the entire material world is also divided into the system, which is the object of research, and its environment (OS). In this case, the energy of such an ("expanded") system not only disappears in one part (system) and appears in another (OS) but is transferred across the boundaries of the system by various energy carriers $\Theta_{j}$ (mass $M$, charges $\Theta_{e}$, moles $N_{k}$ of $k$-th substances in any of their state of aggregation, and the impulses of their chaotic $P_{k}=M_{k} v_{k}$ or ordered $\boldsymbol{P}_{k}=M_{k} \boldsymbol{v}_{k}$ motion). For such a case, the "equation of motion of energy in bodies" is valid, proposed back in 1874 by the Russian professor N. Umov [11]:

$d U / d t+\oint_{j_{u}} \cdot d \mathbf{f}=0$

where $\boldsymbol{j}_{u}\left(\mathrm{~W} \mathrm{~m}^{-2}\right)$ is the internal energy flux density through the vector element $d \boldsymbol{f}$ of the closed surface $f$ of the system with volume $V$ in the direction of the external normal $\boldsymbol{n}$. This form of the law of conservation of energy takes into account the kinetics of real processes, without making any assumptions about the mechanism of energy transfer and the internal structure of the system. In this case, the flux $\boldsymbol{j}_{u}$ consists of the flux $\boldsymbol{j}_{u j}$ of the energy of the $j$-th kind $U_{j}$, carried by independent energy carriers $\Theta_{j}$. For simplicity, we will consider the case of fixed boundaries and constant volume $V$. Then each of these fluxes $\boldsymbol{j}_{u j}$ is expressed by the product of the flux $\boldsymbol{j}_{j}$ of the energy carrier $\Theta_{j}$ by its potential $\psi_{j}=d U_{j} / d M$ (specific energy), i.e., $\boldsymbol{j}_{u j}=\psi_{j} \boldsymbol{j}_{j}=\psi_{j} \rho_{j} \boldsymbol{v}_{j}$, where $\boldsymbol{v}_{j}$ is the rate of transfer of the $j$-th energy carrier $\Theta_{j}$ through the fixed boundaries of the system, and $\rho_{k}(\boldsymbol{r}, t)=\partial \Theta_{k} / \partial V$ is its density, considered as a function of the field point (radius vector $r$ and time $t$ :

$\boldsymbol{j}_{u}=\Sigma_{j} \boldsymbol{j}_{u j}=\Sigma_{j} \psi_{i} \boldsymbol{j}_{j}$

Using the Gauss-Ostrogradsky theorem, we transform expression (3) to the form $d U / d t+\int \nabla \cdot \mathbf{j}_{u} d V=0$. Then, after decomposing $\nabla\left(\psi_{j} \boldsymbol{j}_{j}\right)$ into independent components $\psi_{j} \nabla \cdot \mathbf{j}_{j}+$ $j_{j} \cdot \nabla \psi_{j}$ the energy conservation law (1) will appear in the form:

$d U / d t+\Sigma_{j} \int \psi_{j} \nabla \cdot \dot{j}_{j} d V+\Sigma_{j} \int j_{j} \cdot \nabla \psi_{j} d V=0(\mathrm{~W})$

${ }^{1}$ Their application is allowed only at the final stage of the application of the theory to a specific object as a kind of conditions for unambiguity.
If we now introduce the concept of the thermodynamic force $\boldsymbol{x}_{j}=-\nabla \psi_{j}$ as a measure of the local field strength of the potential $\psi_{j}$ and, in accordance with the mean value theorem, take outside the integral sign some averaged value of this potential $\Psi_{j}$ for the system as a whole, as well as some averaged value $\boldsymbol{X}_{j}$ of thermodynamic forces $\boldsymbol{x}_{j}$, then equation (3) will take the form:

$d U / d t=\Sigma_{j} \Psi_{j} J_{j}-\Sigma_{j} \boldsymbol{X}_{j} \cdot \boldsymbol{J}_{j}$

where $J_{j}=\oint \boldsymbol{j}_{j} \cdot d \boldsymbol{f}=\int \nabla \cdot \mathbf{j}_{j} d V$ is the scalar flow of the $j$-th energy carrier $\Theta_{j}$ through the boundaries of the system, which has the meaning of its consumption; $\boldsymbol{J}_{j}=\int_{j} d V=\Theta_{j}$ is the vector flow of this energy carrier for the system as a whole, which has the meaning of its momentum.

This (short-range) form of the energy conservation law contains additional parameters of the inhomogeneous (internally nonequilibrium) state $\boldsymbol{x}_{j}$ and the nonequilibrium process $\boldsymbol{j}_{j}$, which were absent in equilibrium thermodynamics. They indicate that the total energy of the system is subdivided not only into external $E$ and internal $U$ (respectively, depending and not dependent on the motion and position of the system relative to the surrounding bodies and fields), and not only into kinetic and potential (dependent and independent of the momentum $\rho v$ of elements $d V$ of its volume $V$ ), but also on the "partial" energies of all its independent energy carriers $U_{j}=\int \rho_{j} \psi_{j} d V$, and $U=\Sigma_{j} U_{j}$. Moreover, each of these forms of energy can change both as a result of the transfer of this energy carrier $\Theta_{j}$ across the boundaries of the system $\left(d_{e} \Theta_{j}\right)$, and as a result of the transformation of other forms of energy into it, i.e., the appearance of its internal sources or sinks $\left(d_{i} \Theta_{j}\right)$. It is more convenient to express this circumstance by the integral balance equation of an arbitrary field quantity $\Theta_{j}[14]$ :

$d \Theta_{j} / d t=-\int \nabla \cdot \dot{j}_{j} d V+\int \sigma_{j} d V=d_{e} \Theta_{j} / d t+d_{i} \Theta_{j} / d t$

where $\int \nabla \cdot \mathbf{j}_{j} d V=J_{j}=-d_{e} \Theta_{j} / d t ; \sigma_{j}$ is the density of sources or sinks of the energy carrier $d_{i} \Theta_{j} / d t==\int \sigma_{j} d V$.

Substituting $\int \nabla \cdot \mathbf{j}_{j} d V$ from (5) into (3), we find:

$d U / d t=\Sigma_{j} \Psi_{j} d \Theta_{j} / d t-\Sigma_{j} \int \psi_{j} \sigma_{j} d V+\Sigma_{j} \int \boldsymbol{j}_{j} \cdot \boldsymbol{x}_{j} d V=0$

Since with the establishment of equilibrium $\left(\boldsymbol{x}_{j}=0\right)$ internal sources or sinks of any energy carriers $\sigma_{j}$ disappear, and $\Psi_{j}=\psi_{j}$, then in equilibrium systems:

$d U=\Sigma_{j} \psi_{j} d \Theta_{j}$

It follows that the last two sums (6) mutually compensate each other:

$\Sigma_{j} \psi_{j} \sigma_{j}=\Sigma_{j} \boldsymbol{j}_{j} \square \boldsymbol{x}_{j}\left(\mathrm{~W} \mathrm{~m}^{-3}\right) ; \Sigma_{j} \Psi_{j} d \Theta_{j} / d t=\Sigma_{j} \boldsymbol{X}_{j} \cdot \boldsymbol{J}_{j}(\mathrm{~W})$ 
We arrive at the same result by applying (4) to an "extended" isolated system (including the environment), for which the flows $J_{j}$ and $J_{j}$ become internal, and $d U / d t=0$. According to this expression, in the process of relaxation of nonequilibrium $\left(\Sigma_{j} \boldsymbol{j}_{i} \cdot \boldsymbol{x}_{i}>0\right)$ both sources $\left(\sigma_{j}>0\right)$ and sinks $\left(\sigma_{i}\right.$ $<0)$ of various energy carriers can arise. In particular, such sources or sinks are available for the number of moles $N_{k}$ of any $k$-th substance that appears or disappears in the course of chemical reactions. It is also known that in the processes of cutting metals or crushing materials, the amount of released heat of dissipation $Q^{d}$ is always less than the expended work $W$ due to the transfer of part of the ordered energy to other forms of their internal energy. At present, this is taken into account in calculations with a "heat output coefficient" less than one. In the general case, however, such sources can be found in all the so-called "emergent" properties of the system, that is, in the new degrees of freedom acquired by it in the process of evolution.

Thus, the equation for the connection between sources and sinks (8) is more general than the principle of increasing entropy in classical thermodynamics. According to him, the emergence of new properties in a system and its evolution is possible only in locally nonequilibrium systems $\left(\boldsymbol{x}_{j} \neq 0\right)$ and is due to the interconversion of its internal energy $U_{j}$ components. In view of its extreme importance, it is expedient to call this provision the "principle of transformation."

Thus, relation (8) reveals the inconsistency of the attempts of R. Clausius and his followers to make the entropy $S$ a "scapegoat" for dissipation and "any irreversibility'. It is easy to see that the substitution of the sum $\Sigma_{j} \Psi_{j} \sigma_{j}$ by the dissipative function $T \sigma_{s}=d_{i} Q / d t$ leads to violation of relation (8) and to the transition of the first principle of classical thermodynamics $d U=d Q-d W$ into the inequality ${ }^{2} T d S>d U$ $+d W[2]$.

These inequalities give rise to a number of problems, ranging from "the question that remains open about the reasons for the monotonic increase in entropy" [12] and the physical meaning of this parameter [13], to the "theory of thermal death of the Universe" [2]. This becomes especially relevant in connection with the obvious inconsistency of the interpretation of entropy as a measure of the probability of a state from the standpoint of thermokinetics, since its fundamental concept of "flow" in relation to entropy is devoid of any meaning. In thermokinetics, this interpretation is replaced by the understanding of entropy as a "thermal impulse" $S=M \bar{v}$ - the momentum of particles that has lost its vector nature due to the chaotic nature of thermal motion [13]. It also becomes obvious that the occurrence of the problem of thermodynamic inequalities can be prevented by passing to the description of the external energy exchange of a nonequilibrium system directly through the scalar $J_{j}$ and vector $\boldsymbol{J}_{j}$ energy carrier fluxes through the boundaries of the system, as follows from Eq. (4). This equation already includes relation (7) and therefore remains valid for nonstatic (irreversible) processes. It is also important that they already contain the required flows $\boldsymbol{J}_{j}$ and forces $\boldsymbol{X}_{j}$, giving them an unambiguous definition and a very specific meaning. This not only makes it superfluous to compose cumbersome and complex equations for the balance of $k$-th substances, charge, momentum, its moment, energy and entropy, but also eliminates the arbitrariness in dividing their product into factors, which changes its value and distorts the contribution of each process to the total rate of energy dissipation.

It is easy to see that this equation unites the laws of conservation of energy used by classical thermodynamics (the first sum) and other fundamental disciplines (the second sum), that is, it unites them into a single law of conservation and conversion of energy (doing work $d W_{j}^{e}=\boldsymbol{X}_{j} \cdot \boldsymbol{J}_{j} d t$ ). Complemented by the uniqueness conditions, which include the equations of state $\Psi_{j}=\Psi_{j}\left(\mathrm{I}_{j}\right)$ and the transport $\boldsymbol{J}_{j}=\boldsymbol{J}_{j}\left(\boldsymbol{X}_{j}\right)$, it allows solving a wide range of problems related to the transfer and transformation of not only heat, but also any other forms of energy.

At the same time, Eq. (4) reveals the need to introduce extensive state parameters coupled with the thermodynamic forces $\boldsymbol{X}_{j}$ in the same way as $\Theta_{j}$ - with the potentials $\psi_{j}$. Such parameters are additional variables of the nonequilibrium state $\boldsymbol{Z}_{j}$, the time derivatives of which $d \boldsymbol{Z}_{j} / d t$ determine the energy carrier fluxes in the system as a whole $\boldsymbol{J}_{j}=d \boldsymbol{Z}_{j} / d t=\Theta_{j}$ $\overline{\boldsymbol{v}}_{j}$. The meaning of these variables is easy to establish if we pay attention to the position $\boldsymbol{R}_{j}$ of the center of the quantity $\Theta_{j}$ in the current and initial (homogeneous) state $\boldsymbol{R}_{j o}$ with density $\bar{\rho}_{j}=\Theta_{i} / V$, which are determined by the well-known relations $\boldsymbol{R}_{k}=\Theta_{j}^{-1} \int_{\rho_{j}} \boldsymbol{r} d V ; \boldsymbol{R}_{j o}=\Theta_{j}^{-1} \int \bar{\rho}_{j} \boldsymbol{p} d V$, where $\boldsymbol{r}$ is the running (Euler) coordinate of the position in space. This immediately implies the existence of parameters

$\boldsymbol{Z}_{m}=\mathrm{I}_{j}\left(\boldsymbol{R}_{j}-\boldsymbol{R}_{j o}\right)=\int\left(\rho_{j}-\bar{\rho}_{j}\right) \boldsymbol{r} d V$

called in thermokinetics "moments of energy carrier distribution" $\Theta_{j}$. They characterize the deviation $\Delta \boldsymbol{R}_{j}=\boldsymbol{R}_{j}$ $\boldsymbol{R}_{j o}$ of the position of the center of the energy carrier in a homogeneous system from its position at equilibrium $\boldsymbol{R}_{j o}=0$ in the process of its redistribution with an average speed $\overline{\boldsymbol{v}}_{j}=d \boldsymbol{R}_{j} / d t$. Due to their introduction, the energy of the system $U$ becomes a function of not only the amount of energy carriers $\Theta_{j}$, but also their position in space $U=\Sigma_{j} U_{j}$ $\left(\Theta_{j}, \boldsymbol{R}_{j}\right)$. This allows us to express the energy conservation law (4) through the parameters of the system as a whole, as is customary in classical thermodynamics.

However, the main advantage of this approach is that it allows one to obtain the main content of the TIP without resorting to any hypotheses, postulates, and considerations

\footnotetext{
2 The sign of the incomplete differential "đ" emphasizes that the elementary amounts of heat $d Q$ and work $d W$ depend on the nature of the process.
} 
of a statistical-mechanical nature, while at the same time arriving at a number of non-trivial consequences. To be convinced of this, let us compare TIP in its existing and proposed thermokinetics form.

\section{Theory of irreversible processes as a consequence of energodynamics}

Although the processes of energy dissipation were also considered in the thermodynamics of R. Clausius, the calculation of the rate and power of these processes became possible only with the appearance of L. Onsager's theory [1]. To do this, he used the principle of increasing entropy $d S / d t$ $>0$ and limited himself to adiabatically isolated systems, in which its increase as equilibrium is approached is solely due to the presence of internal sources in it. At the same time, he assumed the existence of some parameters $A_{i}$, characterizing the deviation of the state from equilibrium, and introduced the concept of the generalized rate of the $i$-th relaxation process $J_{i}=d A_{i} / d t$ as a derivative of this parameter with respect to time $t$, calling it "flow" ${ }^{3}$. Next, he introduced the concept of the thermodynamic force $X_{i}=\partial S / \partial J_{i}$ as a derivative of the entropy $S$ with respect to the corresponding flow, understanding by it the cause of the onset of the relaxation process. This made it possible to represent the rate of increase in entropy $d S / d t$ in such a system as a product of dissimilar scalar fluxes $J_{i}$ and forces $X_{i}$, which serve as an extensive and intense measure of the energy dissipation process, respectively:

$d S / d t=\Sigma_{i}\left(\partial S / \partial J_{i}\right) d A_{i} / d t=\Sigma_{i} X_{i} J_{i}$

Thus, thermodynamics regained the ability to predict not only the final result of this or that relaxation process, but also the cause of its occurrence, its direction, speed and power.

Another fundamentally new position of his theory was the "postulate of linearity", according to which each of the relaxation fluxes $J_{i}$ arises under the action of all thermodynamic forces $X_{i}$ in the system, increasing linearly with an increase in any of them [1]:

$J_{i}=\Sigma_{j} L_{i j} X_{j}$

where $L_{i j}$ is a constant coefficient characterizing the conductivity of the system in relation to the $j$-th applied force $X_{j}$ and called by him "phenomenological".

Strictly speaking, neither the linear laws (9) nor the coefficients $L_{i j}$ in them had the status of phenomenological (based on experience), since in all the laws known by that time (thermal conductivity, diffusion, electrical conductivity, viscous friction, etc.) and the driving force of the same name to the flow, and the corresponding coefficients of thermal conductivity, diffusion, electrical conductivity, viscous friction, etc., were not constant values. Moreover, the very matrix form of Onsager's laws (9) in no way followed from expression (8), according to which, with the disappearance of the flux $L_{i j}$, its contribution to the increase in entropy vanishes at any values of the "foreign" forces $X_{j}$. Nevertheless, his theory made it possible to explain

\footnotetext{
${ }^{3}$ The concept of flux is more suitable for the parameters $\boldsymbol{J}_{i}$
} as time derivatives of the vector analogs $\boldsymbol{Z}_{i}$ of the 94 / Vol. 24 (No. 4) the previously discovered thermomechanical, thermochemical, thermoelectric and other effects by the appearance of additional components $J_{i j}=L_{i j} X_{j}$ of the flux $J_{i}$ $=\Sigma_{j} J_{i j}$, arising under the action of "foreign forces $X_{j}$, that is, by their «superposition». This was very important, since "overlap effects" were by this time widely used in a number of technologies and measurement methods. Suffice it to refer to the thermoelectric effects of Peltier and Seebeck used in thermocouples and refrigeration plants, to the phenomenon of reverse osmosis, which is the basis of modern water desalination technology, to the thermomagnetic Hall effect used in magnetometers, as well as to electromagnetic phenomena that served as the basis for most technologies' XX century. Therefore, Onsager's theory aroused keen interest in researchers from many countries [14-23].

However, since the parameters $A_{i}$ were obviously absent in equilibrium thermodynamics, quasi-thermodynamics remained a kind of formalism. The situation changed when I. Prigogine proposed to find flows and forces from other fundamental disciplines (mechanics, physical chemistry and kinetics, electrodynamics, etc., where they had a vector nature [14]. the concept of entropy was alien, I. Prigogine had to make a number of assumptions, the main one being the hypothesis of local equilibrium, according to which the volume elements of the nonequilibrium continuum $d V$ are in a state of local equilibrium (despite the occurrence of relaxation processes in them), and this state is characterized by the same a set of variables that are in equilibrium (despite the presence of potential gradients), so that the basic equations of these disciplines are valid for them (despite their inevitable transition, like the basic equation of thermodynamics, to inequality). responsible for the "production of entropy" in the system. This hypothesis is also used in other fundamental disciplines, which, for this purpose, divide the object of research into an infinite number of elementary volumes.

Further, I. Prigogine had to confine himself to stationary irreversible processes, in which the nonequilibrium state of the system is maintained by external compulsion (external forces $\boldsymbol{X}_{j}$ ). Since the entropy of such systems remained unchanged, he decomposed $d_{i} S / d t$ into two components, one of which he called "entropy production" and connected it with the heat source of dissipation $Q^{\partial}\left(d_{i} S / d t=d Q^{\partial} / d t>0\right)$, and the other $\left(d_{e} S / d t=d Q / d t\right)$ - with the export of heat $Q$ from the system. Although the components $d_{i} S$ and $d_{e} S$ lost the properties of hollow differentials of entropy as a state parameter, this approach ultimately made it possible to find heat sources from the equations of the balance of the external energy of the system.

However, the main disadvantage of TIP was that this theory was based on the principle of increasing entropy and therefore excluded from consideration the reversible component of real processes associated with the performance of useful work. Meanwhile, it is she who is primarily interested in engineers of all specialties, as well as biophysicists and astrophysicists. In this regard, it has become very important to develop such a system for substantiating TIP, which would not exclude from

parameters $A_{i}$, found later in the framework of thermokinetics

Int. Centre for Applied Thermodynamics (ICAT) 
consideration any (reversible or irreversible) part of real processes and at the same time would be available for teaching this discipline within the framework of thermodynamics courses. This possibility is provided by the above theory, called thermokinetics for brevity [5]. Its benefits will become apparent for the reasons outlined below.

\subsection{Finding flows and forces without drawing up balance equations}

The application of the TIP to the solution of specific problems required the preliminary compilation of cumbersome and very complex equations for the balance of mass $M$, charge $\Theta_{e}$, momentum $\boldsymbol{P}$ and its moment $\boldsymbol{L}$ and energy $U$, in order to find on their basis, the value $d_{i} S / d t>0$, and then the conjugate fluxes $\boldsymbol{J}_{i}$ and forces $\boldsymbol{X}_{i}$. This required a thorough knowledge of all the above-mentioned disciplines, which practically ruled out the possibility of teaching consumer goods in technical universities within the framework of conventional thermodynamics courses.

Further, the expansion of $d_{i} S / d t>0$ into factors allowed arbitrariness, as a result of which the flows $\boldsymbol{J}_{i}$ and forces fluxes $\boldsymbol{J}_{i}$ and forces $\boldsymbol{X}_{i}$ acquired different dimensions and different physical meaning. Moreover, since such a partition did not affect the value of their product, the assessment of the contribution of one or another relaxation process to the overall rate of approach of the system to equilibrium turned out to be ambiguous.

All these drawbacks can be avoided by finding the fluxes $\boldsymbol{J}_{i}$ and forces $\boldsymbol{X}_{i}$ not on the basis of the principle of increasing entropy, but on a more general basis of the law of conservation of energy (4). As follows from it, local thermodynamic forces $\boldsymbol{x}_{\mathrm{j}}$ and fluxes $\boldsymbol{j}_{j}$, as well as their "global" values (for the system as a whole) fluxes $\boldsymbol{J}_{i}$ and forces $\boldsymbol{X}_{i}$, can be found directly from the energy conservation law (4) and have a completely unambiguous meaning of the potential field strength $\psi_{j}$ and the generalized rate of the relaxation process of the corresponding form of partial energy. This is all the more important because the value of the product $\boldsymbol{j}_{j} \square \boldsymbol{x}_{j}$, and, consequently, the estimate of the contribution of each $j$-th process to the relaxation rate of the system depends on the method of splitting this product into factors. Therefore, the elimination of the arbitrariness inherent in consumer goods in the choice of flows and forces is of particular importance in the search for ways to reduce the irreversibility of real processes.

No less important is the cardinal simplification that introduces into the understanding of nonequilibrium thermodynamics and the mastery of its methods, which gives the determination of the main characteristics of real processes on the basis of the law of conservation of energy. It greatly facilitates the subsequent study of special disciplines and makes it possible to teach consumer goods at an earlier stage of training ${ }^{4}$.

\footnotetext{
${ }^{4}$ This served as the basis for the Ministry of Higher Education of the Russian Federation to recommend the monograph "Thermokinetics" as a textbook for universities.
}

\subsection{Simplification of the "phenomenological" laws of Onsager}

Postulating his famous "phenomenological" laws (9), L. Onsager believed that all the terms of its right-hand side $X_{i} J_{i}$ are positive, since they were determined on the basis of the rate of increase of the entropy $d S / d t$, which is a purely positive value. This meant an acceleration of the relaxation process when new forces $X_{j}$ appeared in the system. This is where the idea of "synergetism" arose, that is, mutual reinforcement (cooperation) of heterogeneous processes. Meanwhile, this very idea of the enhancement of the flux $J_{i}$ under the influence of "alien" forces $X_{j}$ contradicts expression (10), from which it follows that when the force $X_{i}$ of the same name is reversed, the contribution of the flux $J_{i}$ to the "production" of entropy $d S / d t$ disappears, no matter what value reached this stream under the influence of "alien" forces $X_{j}$. The inconsistency of Onsager's postulate of linearity is also evidenced by the already mentioned laws of heat conduction (Fourier), diffusion (Fick), electrical conductivity (Ohm), filtration (Darcy), viscous friction (Newton), etc., in which the flows of heat, $k$-th matter, charge and impulse had a single driving force:

$\boldsymbol{J}_{i}=-L_{i} \boldsymbol{X}_{i}$

where $\boldsymbol{X}_{i}$ is gradients of temperature, chemical and electrical potential, pressure and velocity; $L_{i}$ - coefficients of thermal conductivity, diffusion, electrical conductivity and friction.

This contradiction with L. Onsager's postulate (11) is resolved if we take into account that in isolated systems the sum of internal forces in their general physical understanding is always zero $\left(\sum_{i} \boldsymbol{F}_{i}=0\right)$. for $\left.i=1,2, n\right)$. This means that, in accordance with Newton's third law, any of the applied forces $\boldsymbol{F}_{i}$ can be expressed as the sum of $n$-1 reaction forces $\boldsymbol{F}_{j}$ of a different, $j$-th kind: $\boldsymbol{F}_{i}=-\Sigma_{n-1} \boldsymbol{F}_{j}$ The connection of these forces with the thermodynamic forces $\boldsymbol{X}_{j}$ is easy to establish based on the expression for the power $d W_{j} / d t=\boldsymbol{X}_{j} \cdot \boldsymbol{J}_{j}$ $=\boldsymbol{F}_{j} \cdot \overline{\boldsymbol{v}}_{j}$. It follows from it that $\boldsymbol{X}_{i}=\boldsymbol{F}_{i} / \Theta_{i}$, and $\boldsymbol{X}_{i}=\boldsymbol{F}_{i} / \Theta_{i}$ that is, thermodynamic forces $\boldsymbol{X}_{i}$ and $\boldsymbol{X}_{j}$ are specific values of forces in their general physical understanding (as internal stresses). Due to this, laws (13) can be represented in pseudolinear form, which differs from Onsager's laws (11) by only one fewer term $(n-1)$ :

$\boldsymbol{J}_{i}=L_{i} \Sigma_{j}\left(\Theta_{j} / \Theta_{i}\right) \boldsymbol{X}_{j}=\Sigma_{j} L_{i j} \boldsymbol{X}_{j}$

Here $L_{i j}=L_{i} \Theta_{j} / \Theta_{i}$ are the coefficients that combine kinetic and thermodynamic factors and therefore depend both on the properties of the conductivity of the system and on the state of the system ${ }^{5}$.

3.3. Thermodynamic substantiation of Onsager reciprocity relations.

${ }^{5}$ The latter explains why the phenomenological coefficients $L_{i j}$ in TIP have no meaning for either one or the other. 
One of the most important provisions of the TIP are the so-called reciprocity relations between the off-diagonal coefficients $L_{i j}$ and $L_{j i}$ in the matrix "phenomenological" laws postulated by L. Onsager (11):

$L_{i j}=L_{j i}$

These relations make it possible to somewhat reduce the number of proportionality coefficients $L_{i j}$ to be determined experimentally, which in equations (9) increased from $\mathrm{n}$ to $n^{2}$. To substantiate these relations, L. Onsager needed: the theory of fluctuations, the principle of microscopic reversibility, the principle of detailed equilibrium and an additional postulate about the linear nature of the laws of decay of fluctuations [1]. All of these provisions limited the proof to states in the immediate vicinity of equilibrium. However, it is even more important that they went beyond thermodynamics, which led to Onsager's definition of his theory as "quasithermodynamics".

Meanwhile, it can be shown that these relations also follow from the law of conservation of energy (4) on the basis of the independence of the mixed derivative of the order of differentiation with respect to the variables $X_{j}$ and $X_{i}$ :

$\partial^{2} U / \partial X_{i} \partial X_{j}=\partial^{2} U / \partial X_{j} \partial X_{i}$

This directly implies the relationship between unlike flows and forces, which we call "differential reciprocity relations" [24]:

$\left(\partial J_{i} / \partial X_{j}\right)=\left(\partial J_{j} / \partial X_{j}\right)$

These relations are applicable to both linear and nonlinear transport laws, assuming the dependence of the coefficients $L_{i j}$ on the parameters of the equilibrium state $\psi_{i}$ and $\Theta_{i}$. Their application to linear Onsager laws (1) directly leads to the symmetry of the matrix of phenomenological coefficients $L_{i j}=L_{j i}$ :

$\left(\partial J_{i} / \partial X_{j}\right)=L_{i j}=\left(\partial J_{j} / \partial X_{j}\right)=L_{j i}$

Their justification shows that these relationships are a consequence of more general reasons than the reversibility of microprocesses. It explains why the symmetry conditions for the matrix $n^{2}$ of the coefficients $L_{i j}$ often turned out to be valid far beyond the limits mentioned above.

\subsection{Finding "blending effects" without applying reciprocity relations}

The diagonal form of the transfer laws (13) allows us to propose a new method for finding the so-called "superposition effects", according to which these effects can be found as a consequence of the onset of incomplete (partial) equilibrium $\left(\boldsymbol{J}_{j}=0\right)$ [7]. It is known that equilibrium in a complex (polyvariant) system is never achieved simultaneously for all degrees of its freedom: first, that kind of equilibrium is achieved that was disturbed by the latter under the influence of external compulsion. Therefore, the flows $\boldsymbol{J}_{j}$ disappear one after another as the force $\boldsymbol{X}_{j}$ that generated them disappears, until complete equilibrium is established in the system.

The specificity of the method based on this is easier to understand by the example of the diffusion of the $k$-th substance in continuous, nonuniform in composition (component concentrations $c_{j}$, temperature $T$, and pressure $p)$. This flow, according to laws (13), has the form:

$J_{k}=-D_{k} \nabla \mu_{k}$

where $D_{k}$ is the diffusion coefficient of the $k$-th substance; $\mu_{k}$ is its chemical potential.

This expression differs from the diffusion law proposed by Onsager himself in the absence of an additional sum $\Sigma_{j} D_{k j} \nabla \mu_{k j}$. If we represent $\nabla \mu_{k}$ in terms of its derivatives with respect to the concentrations $c_{j}$ of independent components, their temperature $T$ and pressure $p$, then equation (18) can take the form:

$\boldsymbol{J}_{k}=-D_{k}\left(\Sigma_{j} \mu_{k j}{ }^{*} \nabla c_{j}+s_{k}{ }^{*} \nabla T+v_{k}^{*} \nabla p\right)$

where $\mu_{k j}{ }^{*} \equiv\left(\partial \mu_{k} / \partial c_{j}\right), s_{k}{ }^{*} \equiv\left(\partial \mu_{k} / \partial T\right), v_{k}^{*} \equiv\left(\partial \mu_{k} / \partial p\right)$.

Three components of the resulting force $\boldsymbol{F}_{k c}=\Sigma_{j} \mu_{k j}{ }^{*} \nabla c_{j}$ on the right side of this expression are responsible for the usual (concentration) diffusion $\boldsymbol{F}_{k c}=\Sigma_{j} \mu_{k j}{ }^{*} \nabla c_{j}$, thermal diffusion $\boldsymbol{F}_{k T}=-s_{k}{ }^{*} \nabla T$ and barodiffusion $\boldsymbol{F}_{k p}=v_{k}{ }^{*} \nabla p$. This allows one to separate the thermodynamic $\mu_{k j}, s_{k}{ }^{*}, v_{k}{ }^{*}$ and kinetic $D_{k}$ factors of multicomponent diffusion and establish a number of additional relationships between them, confirmed experimentally ${ }^{6}[25]$.

As another example, consider an inhomogeneous system divided into two parts by a porous partition. If a temperature difference $(\Delta T \neq 0)$ is created in it, then a gas or liquid flow occurs through the partition $\boldsymbol{J}_{k}=D_{k}\left(s_{k}{ }^{*} \nabla T-v_{k}{ }^{*} \nabla p\right)$, leading, under conditions of incomplete equilibrium $\left(\boldsymbol{J}_{k}=0\right)$, to the occurrence of a pressure difference on both sides of the partition (Feddersen effect, 1873):

$(\Delta p / \Delta T)_{\mathrm{st}}=-q_{k}^{*} / T v_{k}^{*}$

where $q_{k}{ }^{*}=T s_{k}{ }^{*}$ is the so-called heat of transfer of the $k$-th substance.

This phenomenon is now called thermoosmosis. The opposite phenomenon is also known - the appearance of a temperature difference on both sides of the partition when air or other gas is forced through it. Both of these effects have the same nature with the Knudsen effect (1910) - the appearance of a pressure difference in vessels connected by a capillary or a narrow slit and filled with gas of different temperatures, as well as with the Allen and Jones fountain effect (1938) in liquid helium II, consisting in the outflow of helium from a vessel closed with a porous stopper, at the slightest heating. The opposite phenomenon - the occurrence of a temperature difference when creating a pressure
${ }^{6}$ To obtain such results based on the Onsager diffusion equation with the existing experimental means, turned out to be a mathematically incorrect problem [23]. 
difference on both sides of the partition - is called the mechanocaloric effect (Daunt-Mendelssohn).

In the case of systems that initially have the same pressure on both sides of the porous partition $(\Delta p=0)$ and initially the same concentration of the $k$-th substance $\left(\Delta c_{k}=\right.$ 0 ), when a temperature difference $\Delta T$ is created, a concentration difference occurs on both sides of it (the Soret effect, 1881):

$\left(\Delta c_{k} / \Delta T\right)_{\mathrm{st}=}-q_{k}^{*} / T \mu_{k k}$

The opposite phenomenon is also known - the appearance of temperature gradients during diffusion mixing of components, discovered by Dufour in 1872 and bearing his name. In isothermal systems $(\Delta T=0)$, when a pressure difference $\Delta p$ is created on the membrane, the phenomenon of reverse osmosis occurs - the separation of a binary solution with the release of the $k$-th component (usually a solvent) from it. This phenomenon is widely used in water treatment plants. The resulting concentration difference of the $k$-th component is described by the expression:

$$
\left(\Delta c_{k} / \Delta p\right)_{\mathrm{st}}=-v_{k} / \mu_{k k}
$$

These results correspond to those obtained in the framework of TIP [7,9]. However, now they were the result of the imposition of not flows, but forces, as it followed from Newtonian mechanics. At the same time, one did not have to assume the linearity of phenomenological laws, postulate the constancy of the phenomenological coefficient's $L_{i}$ in them, and resort to Onsager's reciprocity relations. All this expands the scope of the TIP applicability to nonlinear systems and states far from equilibrium [7].

However, it is no less important that the aforementioned "superposition effects" receive a completely different explanation not as a consequence of the "minimum entropy production" in stationary states, but as a result of the disappearance of one of the flows as full equilibrium is established in the system. The advantages of the above method for finding the above effects lie not only in its simplicity, but also in the possibility of its application in nonlinear systems far from equilibrium, where the Onsager reciprocity relations are known to be violated.

\section{Thermokinetics as a synthesis of equilibrium and nonequilibrium Thermodynamics}

According to expression (12), in locally equilibrium systems (where $\boldsymbol{x}_{j}=0$ ) there are no internal sources $\sigma_{j}$ of energy carriers $\Theta_{j}$, so $J_{j}=\int \nabla \cdot \dot{j}_{j} d V=-d \Theta_{j} / d t$, and expression (12) takes the form:

$d U / d t=\Sigma_{j} \Psi_{j} d \Theta_{j} / d t$

This equation differs from that used in the equilibrium TIP [19] by replacing local potentials $\psi_{j}$ with averaged $\Psi_{j}$. In an even more particular case of equilibrium (homogeneous) systems $\left(\Psi_{j}=\psi_{j}\right)$ of constant volume $(V=M v=$ const $)$, this expression takes the form of the combined equation of the 1st and 2nd laws of classical thermodynamics of open systems in a form close to the relation Gibbs [2]:

$d U=T d S+p v d M+\Sigma_{k} \mu_{k} d N_{k}$

The generalized potentials $\psi_{j}$ (absolute temperature $\psi_{s} \equiv$ $T$, specific pressure energy $\psi_{p} \equiv p v$ and chemical potentials of $k$ substances $\psi_{k} \equiv \mu_{k}$ ) appearing in this expression are partial derivatives $\left(\partial U / \partial \Theta_{j}\right)$ of the energy of the system $U=$ $U\left(S, M, N_{k}\right)$ by independent parameters $S, M$ and $N_{k}$, which makes it possible to distinguish the heat transfer of the system, its mass transfer and the diffusion of $k$ substances through its boundaries as independent forms of energy exchange between the system and the environment. This imposes the requirement that the entropy $S$ be independent of the system's mass $M$ and its composition under the conditions $M=$ const, which was not and could not be taken into account at the time of R. Clausius. Meanwhile, this circumstance affects the interpretation of this concept as a measure of the probability of a state, in relation to which the concept of a flow has no meaning ${ }^{7}$. This can be said about the conceptual system of classical thermodynamics as a whole, which was formed according to the principle "from particular to general" and therefore could not foresee all subsequent collisions.

The latter also applies to the concept of equilibrium, which is fundamental for thermodynamics. In mechanics, it was understood as the absence of the resulting force $\boldsymbol{X}_{j}$ due to the equality of the forces of action and reaction of the same nature. This condition is also preserved in thermokinetics, which, therefore, does not need the condition of reaching the maximum entropy. The principle of increasing entropy dS / $\mathrm{dt}>0$ also turns out to be superfluous, since moving the system away from the equilibrium state or approaching it characterizes the change in the forces $\boldsymbol{X}_{j}$ much more clearly. Moreover, it becomes more obvious that it is impossible to prove this principle within the framework of equilibrium thermodynamics.

For this purpose, consider an arbitrary thermomechanical system, the internal energy of which $U$ as an extensive quantity is a function of two also extensive arguments: entropy $S$ and volume $V$, i. e. $U=U(S, V)$. Then, considering the entropy $S$ as the inverse function $S=S(U, V)$, we find that in isolated systems $(U, V=$ const), to which this principle belongs, the entropy remains unchanged, like its arguments. This explains why "we do not find in thermodynamics an impeccably rigorous substantiation of thermodynamic inequalities that is not subject to statistics" [24]. As for nonequilibrium systems, where $U=U\left(S, V, \boldsymbol{R}_{j}\right)$, then $S=S(U, V$, $\boldsymbol{R}_{j}$ ), and the mentioned principle becomes easy to prove. However, this is no longer necessary, since the displacement

\footnotetext{
${ }^{7}$ The concept of "thermoimpulse", proposed in thermokinetics instead of the term entropy [7], is free from this drawback.
} 
vectors $\boldsymbol{R}_{j}$ themselves make this in a more visual and quantitatively defined way.

From the standpoint of thermokinetics, "the principles of the excluded perpetual motion machine of the 1st and 2nd kind" also acquire a completely different meaning. It becomes obvious that spatially homogeneous systems $\left(\boldsymbol{X}_{j}, \boldsymbol{x}_{j}\right.$ $=0$ ) cannot perform either external $W^{e}$ or internal $W^{i}$ work, so that the very need for the concepts of a "perpetual motion machine" of the 1st and 2nd kind disappears.

The existence in nonequilibrium systems of the interrelation of sources and sinks of various energy carriers (7) also eliminates the "flagrant contradiction" of thermodynamics with the theory of evolution, noted by I. Prigogine [14]. Indeed, the terms on the right-hand side of (7) can have different signs, so that its left-hand side contains both sources and sinks of various energy carriers. This means that thermokinetics fully allows for the simultaneous occurrence of relaxation processes of some degrees of freedom of a nonequilibrium system, and removal from equilibrium of others, as it happens in the already mentioned processes of active transport and in "conjugated" chemical reactions.

However, the main advantage of thermokinetics is the generalization of nonequilibrium thermodynamics to nonstatic processes of useful (reversible) energy conversion in various types of thermal and non-thermal machines. In this area, thermokinetics makes it possible to obtain a number of fundamentally new provisions of general physical significance.

\subsection{Finding a universal criterion for the efficiency of energy converters}

The introduction of the concept of energy flows allows us to propose a universal criterion for the efficiency of any energy converter as the ratio of the power at the output $N_{j}$ and the input $N_{i}$ of the converter:

$\eta_{N}=N_{j} / N_{i}=\boldsymbol{X}_{j} \cdot \boldsymbol{J}_{j} / \boldsymbol{X}_{i} \cdot \boldsymbol{J}_{i} \leq 1$

This criterion, which we called power efficiency, is equally applicable to thermal and non-thermal, cyclic and non-cyclic machines, forward and reverse cycles, motors and energy "generators", to installations of "direct" and "indirect" energy conversion. This efficiency differs from the so-called "exergy" efficiency, expressed by the ratio of the workable part of the energy at the output and input of the installation, by the exclusion of "transit" energy flows that are not involved in the energy conversion process, as well as by taking into account the kinetics of the energy conversion process.

It is easy to see that the power efficiency $\eta_{N}$ vanishes twice: at "no load" $\left(\boldsymbol{J}_{j}=0\right)$ and in "short circuit" mode $\left(\boldsymbol{X}_{j}=\right.$ $0)$. This means that it also takes into account the mode of operation of energy converters, that is, it most fully reflects the perfection of the installation and the degree to which it realizes the possibilities that the source of ordered energy provides. Moreover, it is also the only possible indicator of perfection in those cases when the thermodynamic concept of efficiency as the ratio of the work performed $W$ to the energy supplied from the energy source $Q$ becomes inapplicable due to the impossibility of separating energy sources and receivers in a continuous medium. These are force fields, chemically reacting media, polarized or magnetized bodies, dissociated or ionized gases. All this makes it an indispensable tool for analyzing the efficiency of not only energy, but also technological installations, and not only artificial, but also natural energy converters created by nature itself. In addition, this efficiency is always less than one, which is especially important due to the fact that many non-specialists operate with the concept of "over-unity" efficiency, confusing this concept with heat transformation coefficients or cooling coefficients, which may exceed one. It is equally important that the power efficiency makes it possible to reveal the unity of the laws of transformation of any form of energy.

\subsection{Finding the general form of the laws of energy conversion}

Classical thermodynamics considers exclusively cyclic machines in which the working fluid performs a closed process. This is due to the very nature of the law of conservation of energy of equilibrium systems (7), in which only the processes of energy transfer through heat exchange, mass transfer, diffusion, electrization, etc. appear $d U_{j}=$ $\psi_{j} d \Theta_{j}$, but there are no terms $X_{j} J_{j}$, responsible for energy conversion. However, in a cyclical process

$d U_{j}=\oint d\left(\psi_{j} \Theta_{j}\right)=\oint d\left(\psi_{j} \Theta_{j}\right)-\oint \Theta_{j} d \psi_{j}=-\oint \Theta_{j} d \psi_{j}$

If we multiply and simultaneously divide the expression $\Theta_{j} d \psi_{j}$ by the displacement $d \boldsymbol{R}_{j}$ of the energy carrier $\Theta_{j}$ and take into account that $d \boldsymbol{R}_{j}=d \boldsymbol{r}$, then we find that in the steady process of energy conversion (when $\nabla \psi_{j}=d \psi_{j} / d \boldsymbol{r}$ ), $\Theta_{j} d \psi_{j} / d t$ $\left.=\left(\Theta_{j} d \boldsymbol{R}_{j} / d t\right) \nabla \psi_{j}=-\boldsymbol{J}_{j} \square \boldsymbol{X}_{j}\right)$. This means that in cyclic energy conversion processes the output power $N_{j}=đ W_{j} / d t=\boldsymbol{J}_{j} \square \boldsymbol{X}_{j}$ can be expressed in terms of flows $\boldsymbol{J}_{j}$ and forces $\boldsymbol{X}_{j}$ in the same way as in "flow" converters of the same form of energy. This is the basis for the synthesis of the method of cycles in classical thermodynamics and the method of flows in thermokinetics.

To verify this, consider an arbitrary system that transforms one ( $i$-th) form of internal energy into another $(j$ th). For such a system, from (4) at $\boldsymbol{J}_{j}=0$, the power balance follows directly:

$\boldsymbol{X}_{i} \cdot \boldsymbol{J}_{i}+\boldsymbol{X}_{j} \cdot \boldsymbol{J}_{j}=0$

According to (27), flows $\boldsymbol{J}_{i}$ and $\boldsymbol{J}_{j}$ of the same sign correspond to oppositely directed forces $\boldsymbol{X}_{i}$ and $\boldsymbol{X}_{j}$, and vice versa, to forces $\boldsymbol{X}_{i}, \boldsymbol{X}_{j}$ of the same sign correspond to oppositely directed flows $\boldsymbol{J}_{i}$ and $\boldsymbol{J}_{j}$. This expression can be given the form:

$J_{i} / X_{j}=-J_{j} / X_{i}$

If we denote the ratio $J_{i} / X_{j}$ by $L_{j j}$, and the ratio $J_{j} / X_{i}$ by $L_{j i}$, then we arrive at the Casimir antisymmetry conditions:

$L_{j j}=-L_{j i}$ 
These Onsager - Casimir reciprocity relations follow from the law of conservation of energy and therefore do not depend on whether the forces $\boldsymbol{X}_{i}$ and $\boldsymbol{X}_{j}$ belong to even or odd functions of time [15], which gives them a more general character. They indicate that for the processes of energy interconversion L. Onsager's laws (11) should be modified taking into account the opposite sign of the coefficients $L_{j j}$ and $L_{j i}$ :

$J_{i}=L_{i j} X_{i}-L_{j i} X_{j}$

$J_{j}=L_{j i} X_{i}-L_{j j} X_{j}$

This form of the equations of energy transformation takes into account the opposite direction of dissimilar forces and flows and therefore have more reason to be called phenomenological (based on experience). In particular, it is well known that during the operation of a welding transformer, with approaching the "short circuit" mode $\left(X_{j}\right.$ $\rightarrow 0)$, the current in the secondary circuit increases $\left(J_{i} \rightarrow\right.$ $\max )$. This circumstance does not depend on the nature of energy carriers, which makes it possible to reveal in principle the unity of the laws of transformation of any forms of energy, contrary to classical thermodynamics.

Thermokinetics, considering any energy converter as a "four-pole", penetrated by two streams of converted and converted energy, does not need to know the "mechanism" of energy conversion and therefore is free from many concepts and hypotheses associated with cycles and equipment necessary for their implementation. This makes it possible to study the laws of energy conversion in their "pure" form, which does not require knowledge of the processes constituting the cycle, their sequence, equipment required for the implementation of such processes, the principles of their operation and schemes of power plants, etc. even before their necessity is substantiated, is the main advantage of thermokinetics. This is especially important in the process of teaching energy specialties, when the laws have not yet been mastered, which have to be substantiated with their help. It also gives thermokinetics undeniable advantages over classical cycle thermodynamics (see note $5)$.

\subsection{Establishment of the unity of the laws of transformation of any forms of energy}

In classical thermodynamics, it is customary to take it for granted that the efficiency of any reversible non-thermal machine is equal to unity, while for a heat engine it is limited by the thermal efficiency of an ideal Carnot machine:

$$
\eta_{t} \equiv 1-Q_{2} / Q_{1}=1-T_{2} / T_{1}<1
$$

where $T_{1}, T_{2}$ are constant temperatures of heat supply and removal $\left(Q_{1}\right.$ and $\left.Q_{2}\right)$ in the heat engine cycle, equal to the absolute temperatures of the heat source and receiver.

Such "discrimination" of heat engines is based on the firm belief that "heat and work are, in principle, unequal" [2]. Meanwhile, with a more general approach from the standpoint of thermokinetics, it turns out that the essence of the matter lies in the nondiscrimination in equilibrium thermodynamics of "energy" and "energy", that is, workable and inoperable forms of energy. Moreover, the very formulation of the problem of converting heat into work is incorrect due to the fact that thermal energy is a form of energy, that is, a function of state, while work $W$ is a function of the process, and not a form of energy. This, in particular, implies a lack of understanding of the fundamental difference between the concept of efficiency in thermodynamics and other disciplines.

The thermal efficiency $\eta_{t}(35)$ is known to depend neither on the properties of its working fluid, nor on the design features of the machine, nor on the mode of its operation. Such efficiencies are usually called absolute. Strictly speaking, this indicator should not be called "efficiency" at all, since it characterizes not the degree of its perfection, but the "degree of convertibility" of the energy $Q_{1}$ received from the heat source, which at $T_{1}=T_{2}$ is equal to zero for a heat engine.

The concept of efficiency of a mechanical, electrical, etc. engine has a different meaning in other fundamental disciplines. Such efficiencies characterize the ratio of the work $W_{i}$ actually performed by the engine to the theoretically possible $W_{i}{ }^{t}$. They take into account losses only in the machine itself and are ideally equal to one. Such efficiencies are called relative. In classical thermodynamics, they are called internal relative $\eta_{o i}$ and are used to assess the excellence of processes, for example, in compressors or turbines. Naturally, the application of the same term "efficiency" to these two fundamentally different concepts causes non-specialists to misunderstand the inefficiency of heat engines.

The case looks completely different if the power efficiency $\eta_{N}(25)$ is used as an efficiency criterion. Its use makes it possible to prove not only the unity of the laws of transformation of any forms of energy, but also to propose a theory of the similarity of power plants, which complements the classical theory of heat engines by analyzing the relationship of this efficiency with their performance (power $N$ ) and operating mode. This is achieved by reducing equations (30.31) to the dimensionless form

$X_{j} / X_{j o}+J_{j} / J_{j k}=1$

where $X_{j o}$ is the values of the driving forces and flows "idle" of the installation (at $J_{j}=0$ ), and $J_{j k}$ is the flow of converted energy in the "short circuit" mode (at $X_{j}=0$ ), also determined on the basis of equations $(30,32)$.

Thanks to the theory of similarity of power plants, it becomes possible to identify the optimal modes of operation of existing power plants, as well as to assess the prospects of their new varieties [27]. All of the above brings the results of thermodynamic analysis closer to reality, promising a real revolution in its methods.

\section{Conclusion}


1. The main disadvantage of the thermodynamics of irreversible processes (TIP) as a general physical theory is its postulative nature and the initial limitation of energy dissipation processes, which is due to its basing on the principle of increasing entropy $d S / d t$.

2. The approach to the construction of nonequilibrium thermodynamics from a more general standpoint of energodynamics reveals the inconsistency of the hypothesis of local equilibrium and dictates the need to introduce additional variables of the nonequilibrium state as functions of the potential gradient $\nabla \psi_{j}$ and displacement vectors of energy carriers $\boldsymbol{R}_{j}$.

3. Finding the main quantities that the TIP operates thermodynamic forces $\boldsymbol{X}_{j}$ and fluxes $\boldsymbol{J}_{j}$ on a more general basis of the law of conservation of energy allows us to create a more general theory that does not exclude from consideration any (reversible or irreversible) component of real processes and locally nonequilibrium systems.

4. The proposed approach, referred to for brevity as thermokinetics, prevents the occurrence of inequalities in the transition to non-static processes, which makes it possible to take into account the irreversibility of real processes by all disciplines operating with the concept of energy.

5. Thermokinetics establishes a previously unknown relationship between sources and sinks of various energy carriers, on the one hand, and the parameters of nonequilibrium, on the other, called the "principle of transformation" generalizing the principle of increasing entropy to other emergent properties of systems.

6. Allows to give a strictly thermodynamic substantiation of all provisions of the TIP, free from the postulates and considerations of molecular-kinetic and statisticalmechanical theories, which extends the scope of its applicability to nonlinear processes and states far from equilibrium.

7. The existence of the resulting $\boldsymbol{F}_{j}$ dissimilar thermodynamic forces $\boldsymbol{X}_{j}$ acting in polyvariant systems refutes Onsager's postulate about the dependence of each of the independent fluxes $\boldsymbol{J}_{i}$ on all forces acting in the system and allows us to find for each of them a single force, with the disappearance of which this process stops.

8. The method proposed by thermokinetics for finding the effects of "overlap" makes it possible to further reduce the number of empirical coefficients from $n(n+1) / 2$ in TIP to $\mathrm{n}$ and give a new explanation of these effects as a result of the onset of partial (incomplete) equilibrium in a polyvariant system due to mutual compensation parts of the forces.

9. The presentation of classical thermodynamics as a consequence of thermokinetics also gives undeniable advantages, giving a solution to the problem of thermodynamic inequalities, a simpler interpretation of entropy, generalizing the principle of increasing entropy and making it easier to understand the principles of the excluded perpetual motion machine of the 1 st and 2 nd kind.

10. Generalization of TIP to the processes of purposeful transformation of various forms of energy in natural and technical systems reveals their fundamental unity and difference from relaxation processes both in relation to their kinetic equations and reciprocity relations in them.

11. The transition to the study of the kinetics of energy conversion processes allows us to offer a universal criterion of their efficiency, expressing the ratio of power at their output and input, and taking into account their performance and operating mode. This efficiency combines the concepts of absolute, relative, internal, exergy, etc. efficiency with refrigeration, etc. coefficients.

12. The unity of the laws of transformation of thermal and non-thermal forms of energy, proved in the framework of thermokinetics, is reflected in the theory of the similarity of energy conversion processes, which allows transferring the results of the analysis of the efficiency of existing power plants to newly designed ones, taking into account the modes of their upcoming operation.

\section{References:}

[1] Onsager L. Reciprocal relations in irreversible processes // Phys. Rev. 1931.237 (14). 405-426; 238 (12). 2265-2279.

[2] Bazarov IP. Thermodynamics. Edn 4. M.: Vysshaya shkola, 1991. (In Russian).

[3] Prigogine I. Time, structure and fluctuations (Nobel lecture in chemistry in 1977). // UFN, 131 (1980). 185-207. (In Russian).

[4] Einstein A. Creative biography. // Physics and Reality. - M.: Nauka, 1985.S. 131-166. (In Russian).

[5] Etkin V.A. Synthesis and new applications of the energy transfer and energy conversion theories. (Summary of thesis for doct. Techn. Sciences. // Moscow: State Techn. Univ. Press, 1998. - 35 pp. (In Russian).

[6] Etkin VA Thermodynamics of nonequilibrium processes of energy transfer and transformation. Saratov: Saratov: Sarat. Gov. Univers., 1991. (In Russian).

[7] Etkin V. Thermokinetics (Synthesis of Heat Engineering Theoretical Grounds). - Haifa, 2010; Etkin VA. Thermokinetics (thermodynamics of nonequilibrium processes of energy transfer and conversion. Togliatti, 1999. (In Russian).

[8] Etkin V. Energodynamics (Thermodynamic Fundamentals of Synergetics). - New York, 2011: Etkin VA. Energodynamics (synthesis of theories of energy transfer and transformation) - St. Petersburg; "Science", 2008. (In Russian).

[9] Etkin VA. Paralogisms of thermodynamics. Saarbrücken, Palmarium Ac. Publ., 2015.

[10] Etkin VA Synthesis of thermostatics and thermokinetics. - Lulu Inc. (USA), 2020.

[11] Umov NA. Ein Theorem über die Wechselwirkung in Endlichen Entfernungen. // Zeitschrift für Mathematik und Physik. 19(1874), H. 2. § 12.

[12] Landau LD, Livshits EM Statistical physics. Moscow: Nauka, 1964. (In Russian).

[13] Etkin VA. The Many Faces of Entropy. // Bulletin of the House of Scientists of Haifa, 11 (2007). 1520. (In Russian).

[14] Prigogine I. Etude Thermodynamique des Phenomenes Irreversibles. Liege, 1947.

[15] Cazimir H. B. G. // Rev. Mod. Rhys. 17(1945).343348.

[16] Denbig K. Thermodynamics of stationary irreversible processes. M.: Publishing house of foreign lit., 1954. (In Russian). 
[17] Meixner I. Thermodynamik der irreversiblen Processe. Aachen. 1954.

[18] De Groot, SR.; Mazur, P. Nonequilibrium Thermodynamics. Amsterdam, 1962.

[19] Gyarmati I. Introduction to Irreversible Thermodynamics. Budapest, 1960.

[20] Haase R. Thermodynamik der Irreversiblen Processe. - Darmstadt, 1963.

[21] Bakhareva IF Nonlinear nonequilibrium thermodynamics. Saratov: Sarat. Gov. Univers., 1967.

[22] Jou D, Casas-Vázquez J, Lebon G (2010), Extended Irreversible Thermodynamics. Edn 4, 2010.

[23] Demirel Y. Nonequilibrium Thermodynamics. Transport and Rate Processes in Physical, Chemical and Biological Systems, 3rd ed., Elsevier, Amsterdam, 2014.

[24] Etkin V.A. Origin of Onsager's reciprocal relations. //Soviet. Journal of Appl. Physics, 4 (1989), 288293 (translated from Bulletin of Russian Acad. Of Science, Siberian Branch. - Engineering, 4 (1989), 52-57).

[25] Krishtal MA., Volkov AI. Multicomponent diffusion in metals. M.: Metallurgy, 1985.

[26] Putilov K.A. Thermodynamics. M.: Science, 1971. (in Russian).

[27] Etkin VA. Similarity Theory of Energy Conversion Processes. // International Journal of Energy and Power Engineering, 8 (1) .2019.4-11. DOI: $10.11648 /$ j. ijepe. 20190801.12 